\title{
Evaluation of Existing Methods in Cervical Cancer Detection and Related Issues
}

\author{
Saravanan.M.S ${ }^{\mathrm{a}, 1}$ and A.Rajesh ${ }^{\mathrm{b}}$ \\ a Professor, Dept of AI, Saveetha School of Engineering, Saveetha Institute of Medical \\ and Technical Sciences, Chennai, India \\ ${ }^{\mathrm{b}}$ AssociateProfessor, Dept of CSE, Vels Institute of Science, Technology and Advanced \\ Studies, Chennai, India
}

\begin{abstract}
In day-to-day life cancer is the severe disease to cure. This paper deals about the cervical cancer and its related issues. The cervical cancer is a preventable disease, but it is one of the second most leading causes of cancer deaths in women. There are two main types of cervical cancer namely squamous cell carcinoma, adenocarcinoma. The major stages of cervical cancer will be dealt by evaluating with clear diagrams and also the major risk factors of cervical cancer are discussed. Finally the various treatment methods of cervical cancer are used to analyze and present the related issues.
\end{abstract}

Keywords. Cervical cancer, women, squamous cell carcinoma, adenocarcinoma, risk factors.

\section{Introduction}

Cervical cancer is a preventable disease, but it is one of the second most leading causes of cancer deaths in women. The primacy cause of cervical cancer is the infection is high risk types of human papilloma virus (HPV). HPV infected through sexual relation in early life and unusual sexual activity. Cervical cancer affected by mainly 2 HPV types are 16 and 18 is the most common cancer affected in cervix. Cervical cancer termed as benign and malignant growth of cells and tissues.

\subsection{Morphology of cervical epithelium}

There are two types of epithelium present in the surface of the cervix. The lining of the surface is found on skin and it is present in inside of the hollow organs [1]. The ectocervix is covered by squamous epithelium and the canal is covered by columnar epithelium shown in the Figure 1. The squamous epithelium contain plump and deep multiple layers like flatter cells. The columnar epithelium is a tall, looks like a single layer of cellsand extends from the portion of ectocervix [2]. It is a thin, fragile than the squamous cells and it contain multiple glands with the lubrication present in the canal [3].The two types of epithelium meet in the junction called Squamous Columnar Junction (SCJ) shown in Figure 2. The junction is sharp line due to thickness of two

\footnotetext{
${ }^{1}$ Sravanan.M.S, Professor, Dept of AI, Saveetha School of Engineering, Saveetha Institute of Medical and Technical Sciences, Chennai, India.;

E-mail: saranenadu@gmail.com
} 


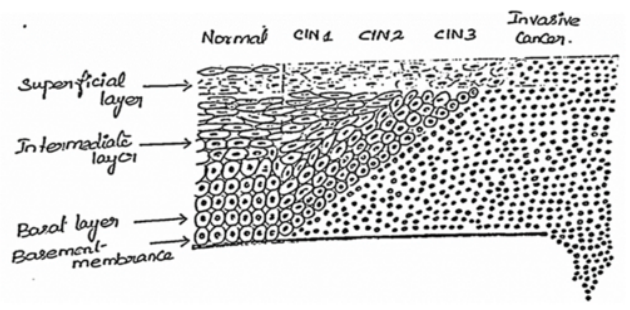

Figure 1. Columnar epithelium changes

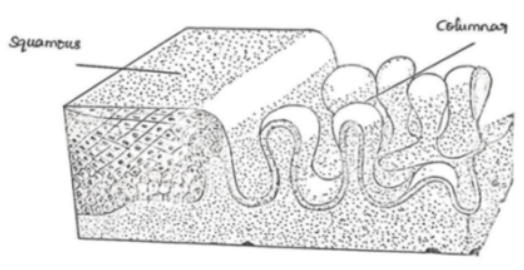

Figure 2.Squamo-Columnar Junction

epithelium layers [4]. This SCJ junction varies depends on woman's age, hormonal, birth, pregnancy status.HPV infection is one of the most common reasons for affecting cervical cancer. Squamous cell cancer is presence in the ectocervix and the affected level of this cancer is $90 \%$. The adenocarcinoma affected in the place of endocervix and the affected level is $10 \%$ [5].The normal cervix consists of exocervix and endocervix. The excocervix is shielded by nonkeratinizing and stratified squamous epithelium [6]. The endocervix is limed with the layer of columnar epithelium and reserve cells are present with complex folding. There are two types of glandular cells namely ciliated and non-ciliated. The ciliated cells are present few and non-ciliated cells are secreting the mucus [7]. For aged person, the metaplastic squamous cells are replaced by the distal part of the cervix. The transformation zone (ectropion) present in the junction of squamous columnar of the exocervix. The ectropion is placed between the squamocolumnar junction and metaplastic squamous epithelium. The immunohistochemistry technique used to observe the endocrine and melanotic cells present in the cervix [8].

\section{Stages of Cervical cancer}

Stages are indicating to evaluate assess the tumor has spread to distant organs of the body. This stages based on physical examination, scanning images and biospies of samples. At stage-1, the cancer is found in the uterus and its spread into the deeper tissue of the cervix. This stage of cancer is not spread to other organs of the body as shown in the figure 3. This stage of cancer is divided into sub stages [9].
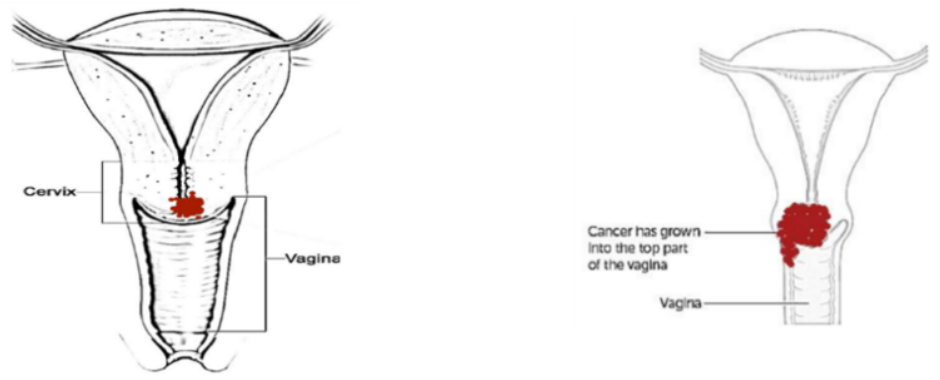

Figure 3. Stage-1 and Stage-2 Cancer

At stage-1A, the invasive cancer is identified by microscope. The stromal invasion measured $5 \mathrm{~mm}$ in depth and not more than $7 \mathrm{~mm}$ width and the depth of the invasion is not more than $5 \mathrm{~mm}$ from the base of normal epithelium [10]. 1A is the invasion of stroma measured about not more than $3 \mathrm{~mm}$ in depth and no more than $7 \mathrm{~mm}$ in wider. 
IB is the tumor size is larger and their lesions constricted to the cervix and the precancer lesions are greater than the stages $1 \mathrm{~A}$. $1 \mathrm{~B} 1$ is the tumor size may be $5 \mathrm{~mm}$ or more than in depth and less than $2 \mathrm{~cm}$ wider. The centimetre size of the tumor is compared to the width of pen or pencil. 1B2 is the tumor size is measured about $2 \mathrm{~cm}$ or more than in depth and the width of the tumor is less than $4 \mathrm{~cm} .1 \mathrm{~B} 3$ is the tumor size is may be $4 \mathrm{~cm}$ or more than in width. At Stage-2, the cancer present inside the area of pelvic and spread behind to nearby area of uterus, vagina and near tissue of the cervix. This tumor is not spread to other organs of the body as shown in the figure 4 .
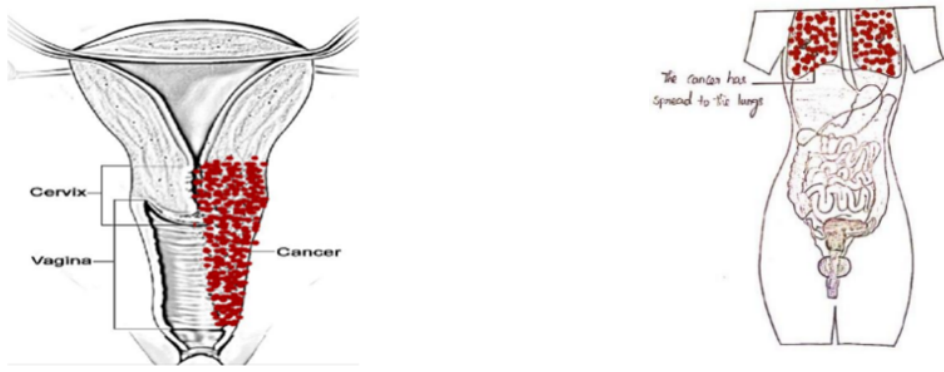

Figure 4. Stage-3 and Stage-4 Cancer

The Stage-2A, the tumor affects the area of upper two-thirds of vagina and it's not spread to the tissue of parametrical area of the cervix. The $2 \mathrm{~A}$, the tumor size is less than $4 \mathrm{~cm}$ wider, $2 \mathrm{~A}$, the tumor size is may be $4 \mathrm{~cm}$ or more than in width. The Stage- $2 \mathrm{~B}$, the cancer spread to the tissue of parametrical area and it does not reach the wall of pelvic. The Stage-3, the tumor affects the mower third part of vagina and spread to the wall of the pelvic. The symptoms of tumor includes kidney swelling (hydronephrosis), syops kidney functioning, region of lymph and there is no spreading of distant organs as shown in figure 4.

\section{Risk factors of Cervical Cancer}

There are various risk factors which increase the chances for developing cervical cancer as shown in table 1. The focus of these factors for women is important to know to do regular Pap test for detecting cervical cancer earlier [10].

Table 1. Factors contributing to cervical cancer

\begin{tabular}{ll}
\hline \multicolumn{1}{c}{ Factors } & Range (\%) \\
\hline Smoking prevalence (\%), women & $2.3[1.7$ to 2.8] \\
Total fertility rate (live births per women) & 2.3 \\
$\begin{array}{l}\text { Hormonal contraception use (\%) (pill, injectable or implant), among } \\
\text { women }\end{array}$ & 3.2 \\
HIV prevalence (\%), adults (15-49 years) & - \\
\hline
\end{tabular}

\section{Treatment of Cervical cancer}

Its aim is to destroy or remove the area affected cervical cancer in the cervix identified as a pre-cancer. There are two methods are ablative or excisional. The ablative method 
is to destroy abnormal tissue by burning or freezing and the excisional methods are bone by surgically to remove abnormal tissues.

\subsection{Cryotherapy}

An ablative method is used to remove the precancerous area present in the cervix by freezing. To apply a cryoprobe (highly cooled metal disc) in the place of cervix and the abnormal or normal areas are freezed and covered. The cryoprobe are contained a tank filled by carbon-dioxide or nitrous oxide gas. This method is performed by health-care [11-15] provider trained in cryotherapy. This technique done by 15 minutes with slight discomfort and it may be performed without anaesthesia.

\subsection{Loop electrosurgical excision procedure (LEEP)}

A loop made of thin wire is used to remove the affected area of the cervix and the wire powered by an electrosurgical unit. The loop cuts and coagulates and using a electrode ball for completing the coagulation. This procedure aim is to destroy the abnormal lesion of the transformation zone. The removal tissue sent to the laboratory for assessing the lession in histopathological examination. This procedure serves for two purposes: remove the lesion and provide the specimen for examining the histopathology. The procedure completed about 30 minutes under local anaesthesia. In this procedure, the patient should wait for few hours or assurance of bleeding does not occur. It is like a surgical procedure and performed by a gynaecologist and it's carried out in the hospitals.

\subsection{Cold knife conization}

This procedure removes the portion of cone-shaped area of the ectocervix and Endocervix. Thremoved depends upon the tissue size of the cancerous lesion and the tissue sent to the laboratory for diagnosis. This procedure is done only in the hospital with health-care trained providers (gynaecologists). This method completed about less than one hour under anesthesia. After procedure, the patient should be discharged to the next day.

\section{Observations}

The observations are listed below as following first it study about the cervical cancer and their symptoms, risk factors and screening methods, Human Papilloma Virus (HPV) is the main reason for the cause of cervical cancer, There are several manual screening techniques for detecting the cervical cancer was observed and the treatment of cervical cancer observes to reduce the infection of cancer affected in the cervix.

\section{Conclusion}

This paper presents the detailed information of cancer disease. The cancer development process sheds light on not only the development of cancer, but later this information will help in creating the feature set proposed here. The cell changes progresses from normal to cancer cell changes its color, texture, appearance, etc., and these visible characteristics are called abnormalities and the staging process and classification processes help in telling the severity of the cancer. The different abnormalities and 
classification of cancer system and the virus responsible of cancer are presented here in detail. This paper gives pre-essential information prior to creating a feature set for an automated system.

\section{References}

[1] Loay, K. Abood, Raad A. Mohammed, Image Segmentation Using Superpixel Based Split and Merge Method.Iraqi Journal of Science, Vol 56, No.1A, pp:233-237, 2015.

[2] Zhi Lu, Gustavo Carneiro, and Andrew P. Bradley, Automated Nucleus and Cytoplasm Segmentation of Overlapping Cervical Cells, PubMed DOI: 10.1007/978-3-642-40811-3_57.

[3] Amanpreetkaur, Ashishvarma, Ssiet, Derabasi, The Marker-aBased Watershed Segmentation- A Review, International Journal of Engineering and Innovative Technology (IJEIT) Volume 3, Issue 3, September 2013.

[4] Kuan Li, Zhi Lu, Wenyin Liu, Jianping Yin, Cytoplasm and nucleus segmentation in cervical smear images using Radiationg GVF Snake, Pattern Recognition Impact Factor: 3.1 Doi: 10.1016/J.Patcog.2011.09.018, April 2012

[5] Marina E.Plissiti and ChristopjorosNikou, Overlapping Cell Nuclei Segmentation Using a Spatially Adaptive Active Physical Model, IEEE Transactions on Image Processing, Vol. 21, No.11, November 2012.

[6] Maykel Orozco-Monteagudo, CosminMihai, HichemSahli and Alberto Taboada-Crispi, Combine Hierarchical Watershed Segmentation and SVM Classification for Pap Smear Cell Nucleus Extraction, Computacion u Sistemas Vol. 16 No.2, pp 133-145, 2012.

[7] RaminMoshavegh, BabkEheshtamiBejnordi, Andrew Mehnert, et al, Automated segmentation offreelying cell nuclei in Pap smears for malignancy-associated change analysis, 34th Annual International Conference of the IEEE EMBS San Diego, California USA, 28TH August- 1st September 2012.

[8] Weimiao Yu, HweeKuan Lee, SrivatsHariharan, Level Set Segmentation of Cellular Images Based on Topological Dependence, Springer-Verlag Berlin Heidelberg G.Bebis et al. (Eds): ISVC 2008, Part I, LNCS 5358, pp. 540-551, 2008.

[9] BalasubramanianGopinath, NatesanShanthi, Automated Segmentation Of ELA Cancer Cells in Microscopic Images for Evaluating the Cytotoxic Effect of Selected Medicina; Plants, Journal of Medical nad Biological Engineering,32(4): 279-289.

[10] MehryarEmambakhsh, HoseinEbrahimnezhad, Mohammad HosseinSedaaghi, Integrated RegiomBased Segmentation using Color Components and Texture Features with Prior Shape Knowledge, Int. J.

[11] Ambeth Kumar.V.D, S. Sharmila, Abhishek Kumar, A. K. Bashir, Mamoon Rashid, Sachin Kumar Gupta \&Waleed S. Alnumay . A novel solution for finding postpartum haemorrhage using fuzzy neural techniques. Neural Computing and Applications (2021) (https://doi.org/10.1007/s00521-020-05683-z)

[12] Hema Kumar.S, J.UdayKiran, V.D.AKumar, G.Saranya, Ramalakshmi V.Effective Online Medical Appointment System.International Journal of Scientific \& Technology Research, Volume 8, Issue 09, September 2019, Pages 803 - 805.

[13] Ambeth Kumar.V.D and S. Sharmila.An Clinical Study on Finding Hypothermia and Postpartum Haemorrhage Using Temperature. International Journal of Psychosocial Rehabilitation, Vol. 24, Issue 05, pp: 2624- 2629, 2020.

[14] S.V. Ruphitha et.al, “ Management of Major Postpartum Haemorrhage by using Zigbee protocol - A Review “, 2021 6th International Conference on Inventive Computation Technologies (ICICT) (DOI: 10.1109/ICICT50816.2021.9358757)

[15] M. Indhumathi et.al , "Healthcare Management of Major Cardiovascular Disease-A review", 2021 6th International Conference on Inventive Computation Technologies (ICICT), (DOI: 10.1109/ICICT50816.2021.9358519) 